\title{
Ultrafast ignition with relativistic shock waves induced by high power lasers
}

\author{
Shalom Eliezer ${ }^{1}$, Noaz Nissim ${ }^{2}$, Shirly Vinikman Pinhasi ${ }^{2}$, Erez Raicher ${ }^{2}, 3$, and José Maria Martinez Val ${ }^{1}$ \\ ${ }^{1}$ Nuclear Fusion Institute, Polytechnic University of Madrid, Madrid, Spain \\ ${ }^{2}$ Applied Physics Division, Soreq NRC, Yavne, Israel \\ ${ }^{3}$ Hebrew University of Jerusalem, Jerusalem, Israel \\ (Received 9 April 2014; revised 11 May 2014; accepted 5 June 2014)
}

\begin{abstract}
In this paper we consider laser intensities greater than $10^{16} \mathrm{~W} \mathrm{~cm}^{-2}$ where the ablation pressure is negligible in comparison with the radiation pressure. The radiation pressure is caused by the ponderomotive force acting mainly on the electrons that are separated from the ions to create a double layer (DL). This DL is accelerated into the target, like a piston that pushes the matter in such a way that a shock wave is created. Here we discuss two novel ideas. Firstly, the transition domain between the relativistic and non-relativistic laser-induced shock waves. Our solution is based on relativistic hydrodynamics also for the above transition domain. The relativistic shock wave parameters, such as compression, pressure, shock wave and particle flow velocities, sound velocity and rarefaction wave velocity in the compressed target, and temperature are calculated. Secondly, we would like to use this transition domain for shockwave-induced ultrafast ignition of a pre-compressed target. The laser parameters for these purposes are calculated and the main advantages of this scheme are described. If this scheme is successful a new source of energy in large quantities may become feasible.
\end{abstract}

Keywords: relativistic hydrodynamics; shock waves; laser; plasma; nuclear fusion; fast ignition

\section{Introduction}

Inertial fusion energy (IFE) is based on high compression ${ }^{[1-3]}$. The reasoning is that it is energetically cheaper to compress rather than to heat and the nuclear reactions are proportional to the density squared. IFE of deuterium-tritium (DT) requires high compression $(>1000)$ and, in particular, the aneutronic fusion ${ }^{[4-6]}$ of protonboron 11 needs extremely high compression $(>10000)$. The high compression is achieved by shock waves and the accumulation of matter during stagnation of the implosion of the target shell.

Shock waves in laser plasma interactions ${ }^{[7]}$ have played an important role in the study of IFE. In 1974 the first direct observation of a laser-driven shock wave was reported ${ }^{[8]}$. A planar solid hydrogen target was irradiated with a $10 \mathrm{~J}$, $5 \mathrm{~ns}$, Nd laser and a pressure of approximately 2 Mbar was measured. Twenty years after this experiment, the Nova laser from Livermore created a pressure of $750 \pm 200 \mathrm{Mbar}^{[9]}$. This was achieved in a collision between two gold foils,

Correspondence to: Shalom Eliezer, Nuclear Fusion Institute, C. Jose Gutierrez Abascal 2, Madrid 28006, Spain. Email: shalom.eliezer@gmail.com where the flyer (Au foil) was accelerated by a high intensity $\mathrm{X}$-ray flux created by the laser-plasma interaction.

In order to achieve nuclear fusion ignition, a mega-joule (MJ) laser with a few nanoseconds pulse duration has been constructed in the USA ${ }^{[10]}$. The central spark ignition of DT is expected in the near future. In this scheme the target and the driver pulse shape are designed in such a way that only a spark at the centre of the compressed fuel is heated and ignited $^{[11,12]}$. The rest of the fuel is heated by $\alpha$ particles produced in the DT reactions.

In order to ignite a DT target with significantly less than a few MJ of energy, it was suggested ${ }^{[13,14]}$ to separate the drivers that compress the target from those that heat the target. This idea is called fast ignition (FI), and triggers not in a central spark, but in a secondary interaction of an igniting driver of a very short duration, such as a multiPetawatt (PW) laser beam. The PW laser is supposed to form a channel for a few picoseconds in the plasma atmosphere and to ignite a part of the fuel at the stagnation point of the implosion. For this purpose it is estimated that ignition requires of the order of a few tens of kilo-joule of laser energy for a duration of approximately $10 \mathrm{ps}$ with an irradiance of the order of $10^{20} \mathrm{~W} \mathrm{~cm}^{-2}$. The FI problem is that the laser pulse does not penetrate directly into the 
compressed target, which has an electron density of the order of $10^{24} \mathrm{~cm}^{-3}$. Therefore many schemes of FI have been suggested: (1) the laser energy is converted into electrons that ignite the target ${ }^{[15]}$. (2) The laser energy is converted into protons that ignite the target ${ }^{[16]}$. (3) Since the heating in the previous proposals is not confined and furthermore it is necessary to avoid preheating, a gold cone ${ }^{[17]}(\mathrm{Au}$ density/solid DT density $\sim 100$ ) is stuck in the spherical pellet. (4) FI is induced by plasma jets ${ }^{[18]}$ that are induced by the same laser system that compresses the pellet. (5) The FI is achieved by a plasma flow created from a thin exploding pusher foil ${ }^{[19,20]}$. (6) Plasma blocks for FI have also been suggested ${ }^{[21,22]}$. (7) Murakami et al. $^{[23]}$ revived the old idea of impact fusion with the help of the cone. (8) The use of clusters ${ }^{[24]}$ was also suggested to ignite the compressed pellet. (9) Furthermore, the use of an extra laser-induced shock wave created by the same lasers that compressed the target in order to ignite the target was suggested ${ }^{[25]}$. (10) Alternatively the FI shock wave from a laser-accelerated impact foil ${ }^{[26,27]}$ was proposed. The shock wave ignition schemes are actually based on heating by collision of two shock waves using tailored laser pulses that had already been suggested ${ }^{[28]}$ even before the idea of FI was explicitly published $^{[13,14]}$.

It is well known that the interaction of a high power laser (HPL) with a planar target creates a one dimensional (1D) shock wave $e^{[29,30]}$. The theoretical basis for laser-induced shock waves analysed and measured experimentally so far is based on plasma ablation. For laser intensities in the range $10^{12} \mathrm{~W} \mathrm{~cm}^{-2}<I_{\mathrm{L}}<10^{16} \mathrm{~W} \mathrm{~cm}^{-2}$ and nanosecond pulse durations a hot plasma is created. This plasma exerts a high pressure on the surrounding material, leading to the formation of an intense shock wave moving into the interior of the target. The momentum of the out-flowing plasma balances the momentum imparted to the compressed medium behind the shock front, similar to a rocket effect.

For $I_{\mathrm{L}}<10^{16} \mathrm{~W} \mathrm{~cm}^{-2}$ the ablation pressure is dominant. For $I_{\mathrm{L}}>10^{16} \mathrm{~W} \mathrm{~cm}^{-2}$ the radiation pressure is the dominant pressure at the solid-vacuum interface and the ablation pressure is negligible. In this last case the ponderomotive force drives the shock wave. For laser irradiances $I_{\mathrm{L}}>$ $10^{21} \mathrm{~W} \mathrm{~cm}^{-2}$ one gets a relativistic laser-induced shock wave $^{[31]}$. The theoretical foundation of relativistic shock waves is based on relativistic hydrodynamics ${ }^{[32]}$ and is first analysed by $\mathrm{Taub}^{[33]}$. Relativistic shock waves may be of importance in intense stellar explosions or in collisions of extremely high energy nuclear particles. Furthermore, relativistic shock waves may be a new route for FI nuclear fusion.

In Section 2 the formalism of relativistic shock waves is given for further consideration. In Section 3 the laserinduced shock wave equations are explicitly written and solved numerically without approximation for the first time. In a recent publication ${ }^{[31]}$ the solution is given only for very strong relativistic shocks, whereas in this paper the transition between relativistic and nonrelativistic laser-induced shock waves is obtained. It turns out that this transition domain is important and relevant for the FI scheme as described in Section 4 . The paper is concluded with a short summary and discussion.

\section{Relativistic shock waves}

The relativistic 1D (or non-relativistic ${ }^{[34]}$ ) shock wave is described by five variables: the particle density $n$ (or the density $\rho=M n$, where $M$ is the particle mass), the pressure $P$, the energy density $e$, the shock wave velocity $u_{\mathrm{s}}$, and the particle flow velocity $u_{\mathrm{p}}$, assuming that we know the initial condition of the target: $n_{0}$ (or $\rho_{0}$ ), $P_{0}, e_{0}$, and the particle flow velocity $u_{0}$, before the shock arrival. The four equations relating the shock wave variables are the three Hugoniot relations describing the conservation laws of energy, momentum, and particles, and the equation of state ${ }^{[35,36]}$ connecting the thermodynamic variables of the state under consideration. In order to solve the problem an extra equation is required, which in our case we derive from a laser-plasma interaction model.

The relativistic hydrodynamic starting point is the energy momentum 4-tensor $T_{\mu \nu}$ given by

$$
T_{\mu \nu}=(e+P) U_{\mu} U_{\nu}+P g_{\mu \nu} .
$$

$U_{\mu}(\mu=0,1,2,3)$ is the dimensionless 4-velocity, where the subscript 0 indicates the time component and subscripts 1,2 , and 3 indicate the space $x, y$, and $z$ components, respectively, and $g_{\mu \nu}$ is the metric tensor,

$$
\begin{gathered}
u_{\mu}=c U_{\mu}=\left(\gamma c, \gamma v_{1}, \gamma v_{2}, \gamma v_{3}\right), \\
g_{\mu \nu}: g_{00}=-1, g_{11}=g_{22}=g_{33}=1, g_{\mu \nu}=0 \text { if } \mu \neq v \\
\gamma=\frac{1}{\sqrt{1-\beta^{2}}} ; \quad \beta=\frac{v}{c} ; \quad v=\sqrt{v_{1}^{2}+v_{2}^{2}+v_{3}^{2}},
\end{gathered}
$$

where $c$ is the speed of light in vacuum and $v$ is the 3dimensional fluid particle velocity. Since our equation (1) is the starting point we write it more explicitly as

$$
\begin{gathered}
T_{00}=\gamma^{2}(e+P)-P \\
T_{0 i}=T_{i 0}=-\gamma^{2}(e+P)\left(\frac{v_{\mathrm{i}}}{c}\right) \quad \text { for } i=1,2,3 \\
T_{i j}=\gamma^{2}(e+P)\left(\frac{v_{\mathrm{i}}}{c}\right)\left(\frac{v_{j}}{c}\right)+P \delta_{i j} \quad \text { for } i, j=1,2,3 .
\end{gathered}
$$

In our 1D model one has for the velocity vector $\mathbf{v}=(v, 0,0)$ and the Lorentz transformation is

$$
\text { Lorentz transformation }=\left(\begin{array}{cccc}
\gamma & -\gamma \beta & 0 & 0 \\
-\gamma \beta & \gamma & 0 & 0 \\
0 & 0 & 1 & 0 \\
0 & 0 & 0 & 1
\end{array}\right) .
$$


Energy-momentum conservation, particle number conservation, and the equation of state (EOS) are given accordingly (Einstein summation is assumed from 0 to 3 for identical indices)

$$
\begin{aligned}
\frac{\partial T_{\mu}^{v}}{\partial x^{\nu}} \equiv \partial_{\nu} T_{\mu}^{v}=0 \quad \text { for } \mu=0,1,2,3 \\
\frac{\partial\left(n U^{\mu}\right)}{\partial x^{\mu}} \equiv \partial_{\mu}\left(n U^{\mu}\right)=0 \\
P=P(e, n) .
\end{aligned}
$$

We use equations (1), (3) and (5) for the conservation of energy density flux $c\left[T_{0 \mathrm{x}}\right]_{0}=c\left[T_{0 \mathrm{x}}\right]_{1}$, the conservation of momentum density flux $\left[T_{\mathrm{xx}}\right]_{0}=\left[T_{\mathrm{xx}}\right]_{1}$, and the conservation of particle number flux $\left[n U_{\mathrm{x}}\right]_{0}=\left[n U_{\mathrm{x}}\right]_{1}$ along the shock wave singularity, with the subscripts 0 and 1 respectively denoting the domains before and after shock arrival, to obtain the following equations

$$
\begin{aligned}
\gamma_{0}^{2} \beta_{0}\left(e_{0}+P_{0}\right) & =\gamma_{1}^{2} \beta_{1}\left(e_{1}+P_{1}\right) \\
\gamma_{0}^{2} \beta_{0}^{2}\left(e_{0}+P_{0}\right)+P_{0} & =\gamma_{1}^{2} \beta_{1}^{2}\left(e_{1}+P_{1}\right)+P_{1} \\
\gamma_{0} \beta_{0} n_{0} & =\gamma_{1} \beta_{1} n_{1},
\end{aligned}
$$

where $\gamma_{\mathrm{i}}$ and $\beta_{\mathrm{i}}=v_{\mathrm{i}} / c$ for the domains 0 and 1 are defined in equation (2), where $v_{0}$ and $v_{1}$ are the inflow and outflow onto the shock wave singularity. Figure 1 describes the fluid flow velocities $v_{0}$ and $v_{1}$ as seen in the shock wave singularity frame of reference $S_{1}$ and the shock wave velocity $u_{\mathrm{s} 1}$ and the particle flow velocities $u_{\mathrm{p} 1}$ and $u_{\mathrm{p} 0}=u_{0}$ as seen in the laboratory frame of reference.

From equations (6) the velocities $v_{0}$ and $v_{1}$ are obtained

$$
\begin{gathered}
\frac{v_{0}}{c} \equiv \beta_{0}=\sqrt{\frac{\left(P_{1}-P_{0}\right)\left(e_{1}+P_{0}\right)}{\left(e_{1}-e_{0}\right)\left(e_{0}+P_{1}\right)}} \\
\frac{v_{1}}{c} \equiv \beta_{1}=\sqrt{\frac{\left(P_{1}-P_{0}\right)\left(e_{0}+P_{1}\right)}{\left(e_{1}-e_{0}\right)\left(e_{1}+P_{0}\right)}},
\end{gathered}
$$

and the relativistic Hugoniot equation is derived ${ }^{[33]}$,

$$
\begin{aligned}
& \frac{\left(e_{1}+P_{1}\right)^{2}}{n_{1}^{2}}-\frac{\left(e_{0}+P_{0}\right)^{2}}{n_{0}^{2}} \\
& =\left(P_{1}-P_{0}\right)\left[\frac{\left(e_{0}+P_{0}\right)}{n_{0}^{2}}+\frac{\left(e_{1}+P_{1}\right)}{n_{1}^{2}}\right] .
\end{aligned}
$$

Assuming that in the laboratory the target is initially at rest, $u_{0}=0$, the shock wave velocity $u_{\mathrm{s}}$ and the particle flow velocity $u_{\mathrm{p}}$ in the laboratory frame of reference are related to the flow velocities $v_{0}$ and $v_{1}$ in the shock wave rest frame of reference by

$$
\begin{aligned}
& u_{\mathrm{s}}=-v_{0}, \\
& u_{\mathrm{p}}=\frac{v_{1}-v_{0}}{1-\frac{v_{0} v_{1}}{c^{2}}} .
\end{aligned}
$$

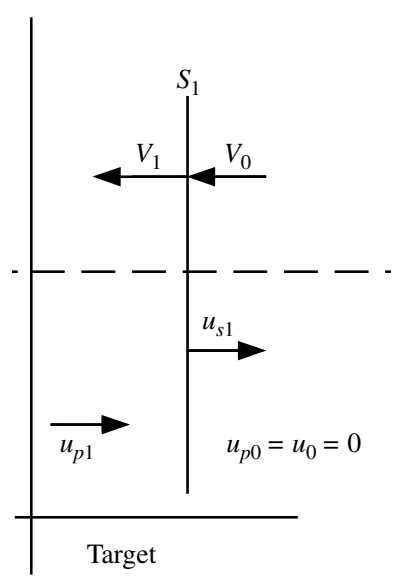

Figure 1. The fluid flow velocities $v_{0}$ and $v_{1}$ as seen in the shock wave singularity frame of reference $S_{1}$ and the shock wave velocity $u_{\mathrm{s} 1}$ and the particle flow velocities $u_{\mathrm{p} 1}$ and $u_{\mathrm{p} 0}=u_{0}$ as seen in the laboratory frame of reference.

The EOS taken here in order to calculate the shock wave parameters is the ideal gas EOS

$$
e=\rho c^{2}+\frac{P}{\Gamma-1},
$$

where $\Gamma$ is the specific heat ratio and $v_{0}$ and $v_{1}$ are given in equations (7) .

\section{Laser-induced shock waves}

This paper analyses the shock wave created in a planar target by the ponderomotive force induced by very high laser irradiance. In this domain of laser intensities the force acts on the electrons that are accelerated and the ions that follow accordingly. This model describes our piston model ${ }^{[37,38]}$ as summarized schematically in Figure 2: Figure 2(a) shows the capacitor model for laser irradiances $I_{\mathrm{L}}$, where the ponderomotive force dominates the interaction. In Figure 2(b) the system of the negative and positive layers is called a double layer (DL), $n_{\mathrm{e}}$ and $n_{\mathrm{i}}$ are the electron and ion densities respectively, $E_{\mathrm{x}}$ is the electric field, $\lambda_{\mathrm{DL}}$ is the distance between the positive and negative DL charges, and $\delta$ is the solid density skin depth of the foil. The DL is geometrically followed by a neutral plasma where the electric field decays within a skin depth and a shock wave is created. The shock wave description in the laboratory frame of reference is given in Figure 2(c). This DL acts as a piston driving a shock wave ${ }^{[39,40]}$. This model is supported in the literature by particle in cell (PIC) simulation ${ }^{[39,41]}$ and independently by hydrodynamic two-fluid simulations ${ }^{[21,22,42]}$. The relativistic shock wave parameters, such as compression, pressure, shock wave and particle flow velocities, and temperature are calculated here for any compression $\kappa=\rho / \rho_{0}>1$ for the first time in the context of relativistic hydrodynamics. In a recent previous paper this was solved only for $\kappa=\rho / \rho_{0}>4$ with $\Gamma=5 / 3$. 

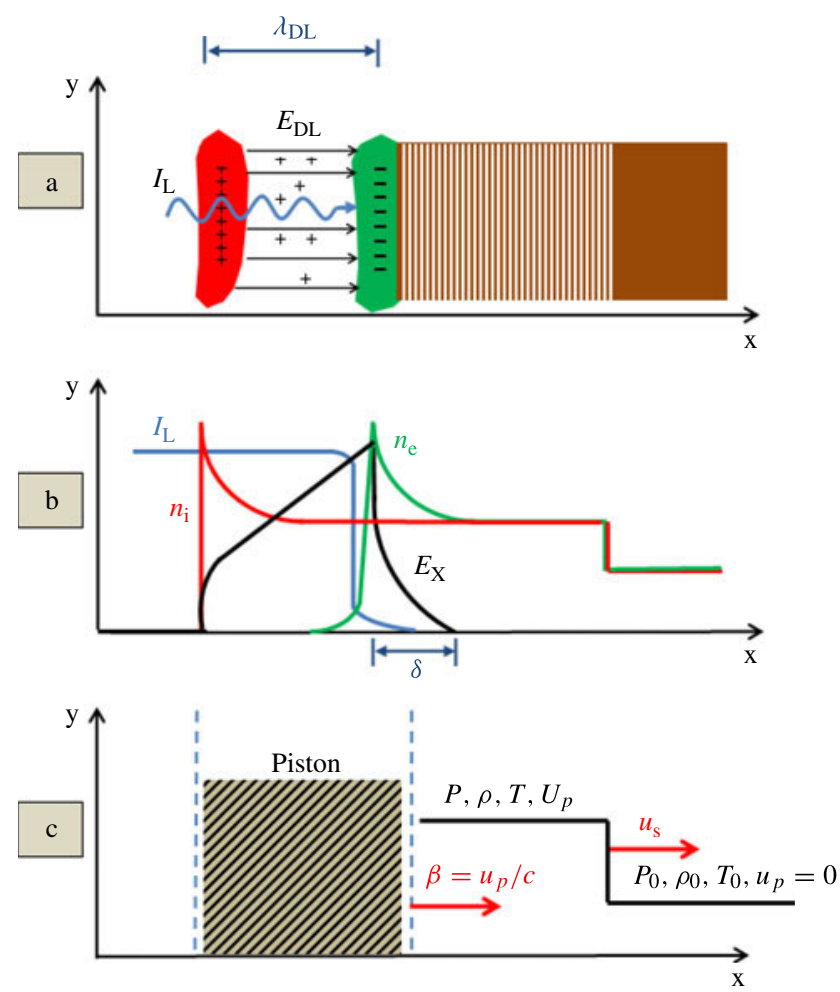

Figure 2. (a) The capacitor model for laser irradiances $I_{\mathrm{L}}$ where the ponderomotive force dominates the interaction. (b) The parameters that define our capacitor model: $n_{\mathrm{e}}$ and $n_{\mathrm{i}}$ are the electron and ion densities accordingly, $E_{\mathrm{X}}$ is the electric field, $\lambda_{\mathrm{DL}}$ is the distance between the positive and negative DL charges. The DL is geometrically followed by a neutral plasma where the electric field decays within a skin depth $\delta$ and a shock wave is created. (c) The shock wave description in the piston model.

For $I_{\mathrm{L}}<10^{16} \mathrm{~W} \mathrm{~cm}^{-2}$ the ablation pressure $P_{\mathrm{a}}$ is dominant and scales with the laser irradiance $I_{\mathrm{L}}$ like $P_{\mathrm{a}} \sim I_{\mathrm{L}}^{\alpha}$, where $\alpha$ is of the order of $2 / 3$ in a $1 \mathrm{D}$ model ${ }^{[7]}$. For $I_{\mathrm{L}}>$ $10^{16} \mathrm{~W} \mathrm{~cm}^{-2}$ the radiation pressure is the dominant pressure at the solid-vacuum interface and the ablation pressure is negligible. In this last case the ponderomotive force drives the shock wave. The equations forrelativistic hydrodynamics with the ideal gas EOS in the laboratory frame of reference are
(i) $\frac{u_{\mathrm{p} 1}}{c}=\sqrt{\frac{\left(P_{1}-P_{0}\right)\left(e_{1}-e_{0}\right)}{\left(e_{0}+P_{1}\right)\left(e_{1}+P_{0}\right)}}$
(ii) $\frac{u_{\mathrm{s} 1}}{c}=\sqrt{\frac{\left(P_{1}-P_{0}\right)\left(e_{1}+P_{0}\right)}{\left(e_{1}-e_{0}\right)\left(e_{0}+P_{1}\right)}}$
(iii) $\frac{\left(e_{1}+P_{1}\right)^{2}}{\rho_{1}^{2}}-\frac{\left(e_{0}+P_{0}\right)^{2}}{\rho_{0}^{2}}$ $=\left(P_{1}-P_{0}\right)\left[\frac{\left(e_{0}+P_{0}\right)}{\rho_{0}^{2}}+\frac{\left(e_{1}+P_{1}\right)}{\rho_{1}^{2}}\right]$

(iv) $\} e_{j}=\rho_{j} c^{2}+\frac{P_{j}}{\Gamma-1} ; j=0,1$.
We have to solve these five equations together with our piston model equation ${ }^{[31,38]}$.

$$
\text { (vi) } P_{1}=\frac{2 I_{\mathrm{L}}}{c}\left(\frac{1-\beta}{1+\beta}\right) ; \quad \beta \equiv \frac{u_{\mathrm{p} 1}}{c} .
$$

Equations (11) and (12) describe six equations with six unknowns: $u_{\mathrm{s}}, u_{\mathrm{p} 1}, P_{1}, \rho_{1}, e_{1}$, and $e_{0}$, assuming that we know $I_{\mathrm{L}}, \rho_{0}, P_{0}, \Gamma$, and $u_{\mathrm{o}}=0$. We take the ideal gas EOS with $\Gamma=5 / 3$. The calculations are conveniently done in the dimensionless units defined by

$$
\begin{aligned}
\Pi_{\mathrm{L}} & \equiv \frac{I_{\mathrm{L}}}{\rho_{0} c^{3}} ; \quad \kappa \equiv \frac{\rho_{1}}{\rho_{0}} ; \\
\kappa_{0} & \equiv \frac{\Gamma+1}{\Gamma-1} ; \quad \Pi=\frac{P_{1}}{\rho_{0} c^{2}} ; \quad \Pi_{0}=\frac{P_{0}}{\rho_{0} c^{2}} .
\end{aligned}
$$

It is important to emphasize that if we take $P_{0}=0$ then we get only the $\kappa>\kappa_{0}$ solutions $^{[31]}$, therefore in order to see the behaviour at the transition between the relativistic and nonrelativistic domains one has to take $P_{0} \neq 0$. In our numerical estimations we take $P_{0}=1 \mathrm{bar}=10^{6}$ in cgs units. For example, the Hugoniot equation (11) (iii) together with the EOS equations $(11)_{(\mathrm{iv})+(\mathrm{v})}$ yield

$$
\begin{aligned}
& \frac{P_{0}}{P_{1}}=\frac{\Pi_{0}}{\Pi}=\mathbf{0} \\
& \Rightarrow\left\{\begin{array}{l}
\Pi=-B\left(\Pi_{0}=0\right)=\frac{(\Gamma-1)^{2}}{\Gamma} \kappa\left(\kappa-\kappa_{0}\right) \\
\kappa \equiv \frac{\rho_{1}}{\rho_{0}} \geqslant \kappa_{0},
\end{array}\right. \\
& \frac{P_{0}}{P_{1}}=\frac{\Pi_{0}}{\Pi} \neq \mathbf{0} \Rightarrow\left\{\begin{array}{l}
\Pi^{2}+B \Pi+C=0 \\
\kappa \equiv \frac{\rho_{1}}{\rho_{0}} \geqslant 1
\end{array}\right. \\
& \Pi=\left(\frac{1}{2}\right)\left(-B \pm \sqrt{B^{2}-4 C}\right) \\
& B=\frac{(\Gamma-1)^{2}}{\Gamma}\left(\kappa_{0} \kappa-\kappa^{2}\right)+\Pi_{0}(\Gamma-1)\left(1-\kappa^{2}\right) \\
& \mathrm{C}=\frac{(\Gamma-1)^{2}}{\Gamma}\left(\kappa-\kappa_{0} \kappa^{2}\right) \Pi_{0}-\kappa^{2} \Pi_{0}^{2} .
\end{aligned}
$$

The compression $\kappa$ as a function of the dimensionless pressure $\Pi=P_{1} /\left(\rho_{0} c^{2}\right)$ is given in Figure 3 for $\kappa_{0}=$ $4(\Gamma=5 / 3)$. Although $P_{0} / P_{1}$ is extremely small one cannot neglect it in the very near vicinity of $\kappa_{0}$ and in this domain one has to solve equation (15) numerically. Furthermore, in order to see the transition between the relativistic and nonrelativistic approximations (see appendix A) one has to solve the relativistic equations with equation (15) in order to see transition effects such as the one shown in Figure 3. However for $\kappa>\kappa_{0}$, for $\left(\kappa-\kappa_{0}\right) / \kappa_{0}>10^{-3}$, the approximation of equation (14) is very good for calculating the shock wave variables as a function of the dimensionless laser irradiance $\Pi_{\mathrm{L}}$.

The numerical solutions of equations (11) and (12) are shown in Figures 4 and 5. Figure 4 gives the dimensionless 


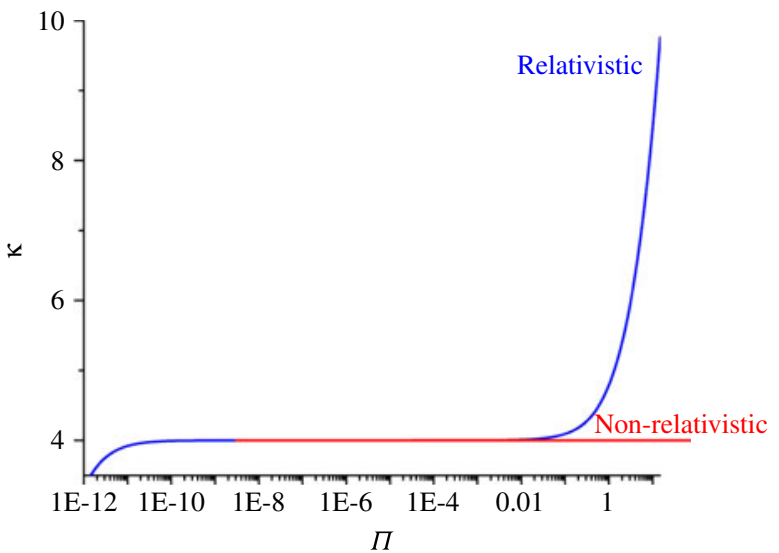

Figure 3. The compression $\kappa=\rho / \rho_{0}$ as a function of the shock wave dimensionless pressure $\Pi=P /\left(\rho_{0} c^{2}\right)$. The numerical values are obtained for $\Gamma=5 / 3$.

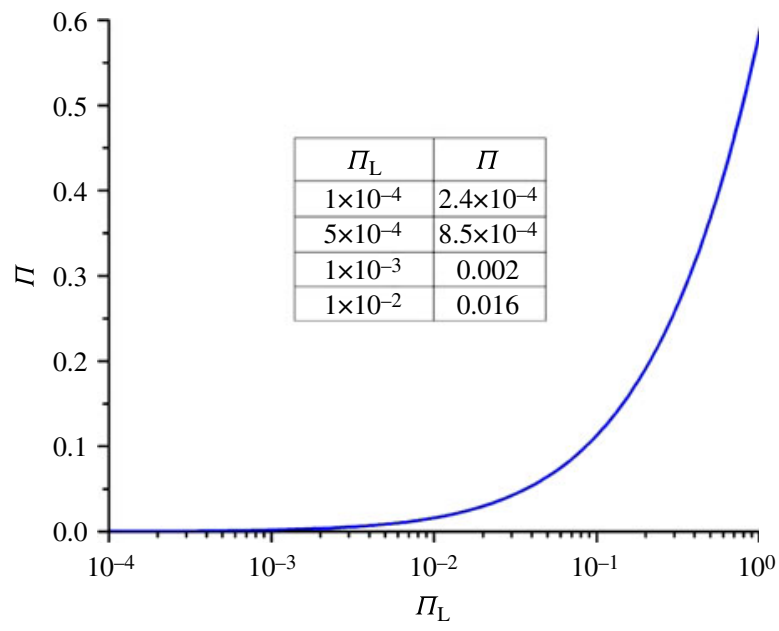

Figure 4. The dimensionless shock wave pressure $\Pi=P /\left(\rho_{0} c^{2}\right)$ versus the dimensionless laser irradiance $\Pi_{\mathrm{L}}=I_{\mathrm{L}} /\left(\rho_{0} c^{3}\right)$ in the range $10^{-4}<$ $\Pi_{\mathrm{L}}<1$. For a better understanding of this graph the inserted table shows numerical values in the range $10^{-4}<\Pi_{\mathrm{L}}<10^{-2}$.

shock wave pressure $\Pi=P_{1} /\left(\rho_{0} c^{2}\right)$ versus the dimensionless laser irradiance $\Pi_{\mathrm{L}}=I_{\mathrm{L}} /\left(\rho_{0} c^{3}\right)$ in the range $10^{-4}<$ $\Pi_{\mathrm{L}}<1$. For a better understanding of this graph and for the practical proposal in the next section, the inserted table shows numerical values in the range $10^{-4}<\Pi_{\mathrm{L}}<10^{-2}$. Figure 5 describes the dimensionless shock wave velocity $u_{\mathrm{s}} / c$ and the particle velocity $u_{\mathrm{p}} / c$ in the laboratory frame of reference versus the dimensionless laser irradiance $\Pi_{\mathrm{L}}=$ $I_{\mathrm{L}} /\left(\rho_{0} c^{3}\right)$ in the range $10^{-4}<\Pi_{\mathrm{L}}<1$ while the inserted table shows numerical values in the range $10^{-4}<\Pi_{\mathrm{L}}<$ $10^{-2}$. As a numerical example we take a target (liquid DT) with initial density $\rho_{0}=0.2 \mathrm{~g} \mathrm{~cm}^{-3}$ irradiated by a laser with intensity $I_{\mathrm{L}}=5 \times 10^{22} \mathrm{~W} \mathrm{~cm}^{-2}$, namely $\Pi_{\mathrm{L}}=$ $9.26 \times 10^{-2}$. In this case our relativistic equations yield a compression $\kappa=\rho / \rho_{0}=4.09$, a pressure $P=2 \times 10^{13}$ bar, a shock wave velocity $u_{\mathrm{s}}=0.35 c$ and a particle velocity $u_{\mathrm{p}}=0.27 c$, where $c$ is the speed of light.

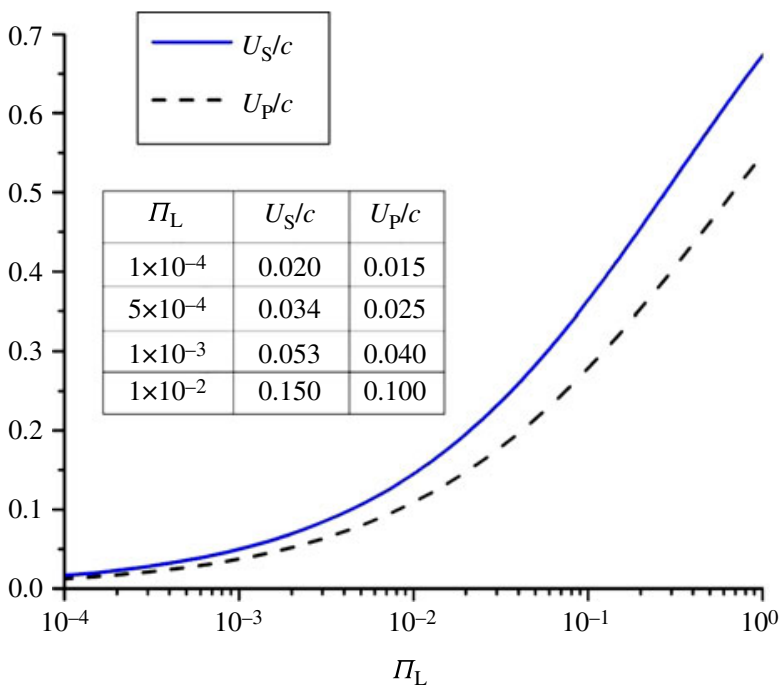

Figure 5. The dimensionless shock wave velocity $u_{\mathrm{s}} / c$ and the particle velocity $u_{\mathrm{p}} / c$ in the laboratory frame of reference versus the dimensionless laser irradiance $\Pi_{\mathrm{L}}=I_{\mathrm{L}} /\left(\rho_{0} c^{3}\right)$ in the range $10^{-4}<\Pi_{\mathrm{L}}<1$. For a better understanding of this graph the inserted tables show numerical values in the range $10^{-4}<\Pi_{\mathrm{L}}<10^{-2}$.

The relativistic speed of sound $c_{\mathrm{S}}$ for an ideal gas EOS is

$$
\frac{c_{\mathrm{s}}}{c}=\sqrt{\left(\frac{\partial P}{\partial e}\right)_{\mathrm{S}}}=\left(\frac{\Gamma P}{e+P}\right)^{1 / 2}=\left[\frac{\Gamma(\Gamma-1) \Pi}{\Gamma \Pi+(\Gamma-1) \kappa}\right]^{1 / 2} .
$$

In the shocked medium the characteristic velocity of a disturbance from the piston to the shock wave front, equal to the rarefaction wave in the shocked medium $c_{\mathrm{rw}}$, is given by

$$
c_{\mathrm{rw}}=\frac{c_{\mathrm{S}}+u_{\mathrm{p}}}{1+\left(\frac{c_{\mathrm{S}} u_{\mathrm{p}}}{c^{2}}\right)} .
$$

Figures 6(a) and 6(b) respectively describe the speed of sound in units of the speed of light, $c_{\mathrm{S}} / c$, and the ratio of shock velocity to the rarefaction velocity, $u_{\mathrm{S}} / c_{\mathrm{rw}}$ as a function of the dimensionless laser irradiance $\Pi_{\mathrm{L}}=$ $I_{\mathrm{L}} /\left(\rho_{0} c^{3}\right)$ in the range $10^{-4}<\Pi_{\mathrm{L}}<1$. The inserted tables show numerical values in the range $10^{-4}<\Pi_{\mathrm{L}}<10^{-2}$.

We now analyse the temperature problem. The partial pressures of an ideal gas that contains electrons and ions with appropriate densities $n_{\mathrm{e}}$ and $n_{\mathrm{i}}$ and temperatures $T_{\mathrm{e}}$ and $T_{\mathrm{i}}$ are $P_{\mathrm{e}}$ and $P_{\mathrm{i}}$, and can be described by

$$
P_{\mathrm{e}}=n_{\mathrm{e}} k_{\mathrm{B}} T_{\mathrm{e}} ; \quad P_{\mathrm{i}}=n_{\mathrm{i}} k_{\mathrm{B}} T_{\mathrm{i}}
$$

If the associated photons in this system are in thermal equilibrium then a radiation temperature $T_{\mathrm{r}}$ can be defined, with a radiation pressure $P_{\mathrm{r}}$ given by ${ }^{[35]}$ 


$$
\begin{aligned}
& P_{\mathrm{r}}=(1 / 3) a T_{\mathrm{r}}^{4} ; \\
& a=\left(\frac{1}{15}\right)\left(\frac{k_{\mathrm{B}}^{4}}{h^{3} c^{3}}\right)=7.56 \times 10^{-15}\left[\mathrm{erg}\left(\mathrm{cm}^{-3} \mathrm{~K}^{-4}\right)\right]
\end{aligned}
$$

For a plasma in local thermal equilibrium satisfying $T_{\mathrm{e}}=$ $T_{\mathrm{i}}=T_{\mathrm{r}}=T$, where the ions have an ionization $Z$ and an atomic number $A$, implying a ion mass of $A m_{\mathrm{p}}$, where $m_{\mathrm{p}}$ is the proton mass, the plasma pressure is given by

$$
P=P_{\mathrm{i}}+P_{\mathrm{e}}+P_{\mathrm{r}}=(Z+1) n_{\mathrm{i}} k_{\mathrm{B}} T+\left(\frac{1}{3}\right) a T^{4} .
$$

If the ion density satisfies

$$
n_{\mathrm{i}}\left[\mathrm{cm}^{-3}\right] \ll 1.56 \times 10^{27}\left(\frac{k_{\mathrm{B}} T}{m_{\mathrm{e}} c^{2}}\right),
$$

then the radiation pressure is dominant and the temperature is given by

$$
T \approx\left(\frac{3 P}{a}\right)^{1 / 4}
$$

It is conceivable to assume that electrons and ions are in thermal equilibrium, i.e., $T_{\mathrm{e}}=T_{\mathrm{i}}$, however the shocked area is not optically thick for the energetic photons. In this case the energetic photons created by bremsstrahlung leave the system, implying $T_{\mathrm{r}} \ll T_{\mathrm{e}}$, or one can have a situation where radiation temperature is not defined at all. Therefore if the photon radiation in equation (19) is negligible then one has

$$
k_{\mathrm{B}} T=m_{\mathrm{p}} c^{2}\left(\frac{A}{Z+1}\right)\left(\frac{\Pi}{\kappa}\right) .
$$

Therefore in general we can write that the plasma temperature is constrained to the following range

$$
\left(\frac{m_{\mathrm{p}} c^{2}}{k_{\mathrm{B}}}\right)\left(\frac{A}{Z+1}\right)\left(\frac{\Pi}{\kappa}\right)>T>\left(\frac{3 P}{a}\right)^{1 / 4} .
$$

Taking the example given above for liquid DT with $A=$ 2.5, $Z=1, m_{\mathrm{p}}=938.3 \mathrm{MeV} / c^{2}$ and initial density $\rho=$ $0.2 \mathrm{~g} \mathrm{~cm}^{-3}$ irradiated by a laser with intensity $I_{\mathrm{L}}=5 \times$ $10^{22} \mathrm{~W} \mathrm{~cm}^{-2}$, namely $\Pi_{\mathrm{L}}=9.26 \times 10^{-2}$, we get $\Pi=$ $0.11, \kappa=4.09$ and a temperature in the range $26.2 \mathrm{keV}<$ $k_{\mathrm{B}} T<31.6 \mathrm{MeV}$. However, for $k_{\mathrm{B}} T>1 \mathrm{MeV}$ we have electron-positron pair production ${ }^{[43,44]}$ and new physics is required here for the temperature calculations. It is out of the scope of this paper to analyse this exotic case here.

\section{An ultrafast ignition solution to the energy problem}

In order to solve the energy problem of future generations scientists have considered using controlled nuclear fusion energy. One of the approaches is the well-known inertial confinement fusion driven by HPLs where the physics is based on compressing and igniting rather than confining the
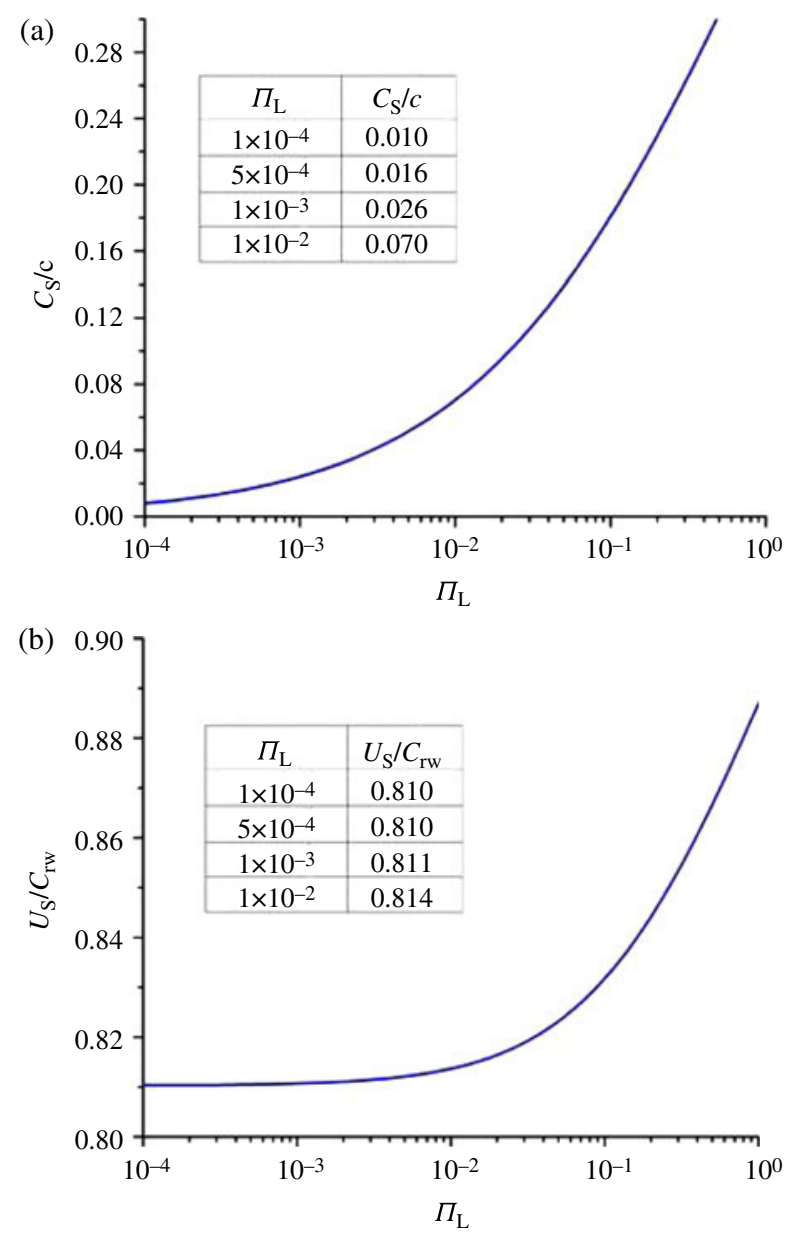

Figure 6. The speed of sound $c_{\mathrm{S}}$ is given in units of the speed of light $c$ in (a) and the ratio of the shock velocity to the rarefaction velocity, $u_{\mathrm{S}} / c_{\mathrm{rw}}$ is shown in (b) as function of the dimensionless laser irradiance $\Pi_{\mathrm{L}}=I_{\mathrm{L}} /\left(\rho_{0} c^{3}\right)$ in the range $10^{-4}<\Pi_{\mathrm{L}}<1$. The inserted tables show numerical values in the range $10^{-4}<\Pi_{\mathrm{L}}<10^{-2}$.

fuel ${ }^{[1,2]}$. In order to ignite the fuel with less energy it was suggested to separate the drivers that compress the target from whose that ignite the target ${ }^{[13,14]}$. First the fuel is compressed, then a second driver ignites a small part of the fuel while the alpha particles created in the DT interaction heat the rest of the target. This idea is called FI. The problem with FI is that the laser pulse does not penetrate directly into the compressed target; therefore many alternative schemes have been suggested ${ }^{[45]}$.

The laser solution of the energy problem requires very sophisticated high power laser science and engineering (HPLSE). In a recent paper ${ }^{[46]}$ the various HPLSE optimizations and design constraints for a laser fusion power plant are beautifully summarized and analysed. From the many possible proposals to solve the energy problem with HPLs we consider three criteria for choosing the best candidate (present or future): (i) Understanding the physics. In HPLtarget interactions there are many scientific problems not yet fully understood, such as laser-plasma instabilities, 


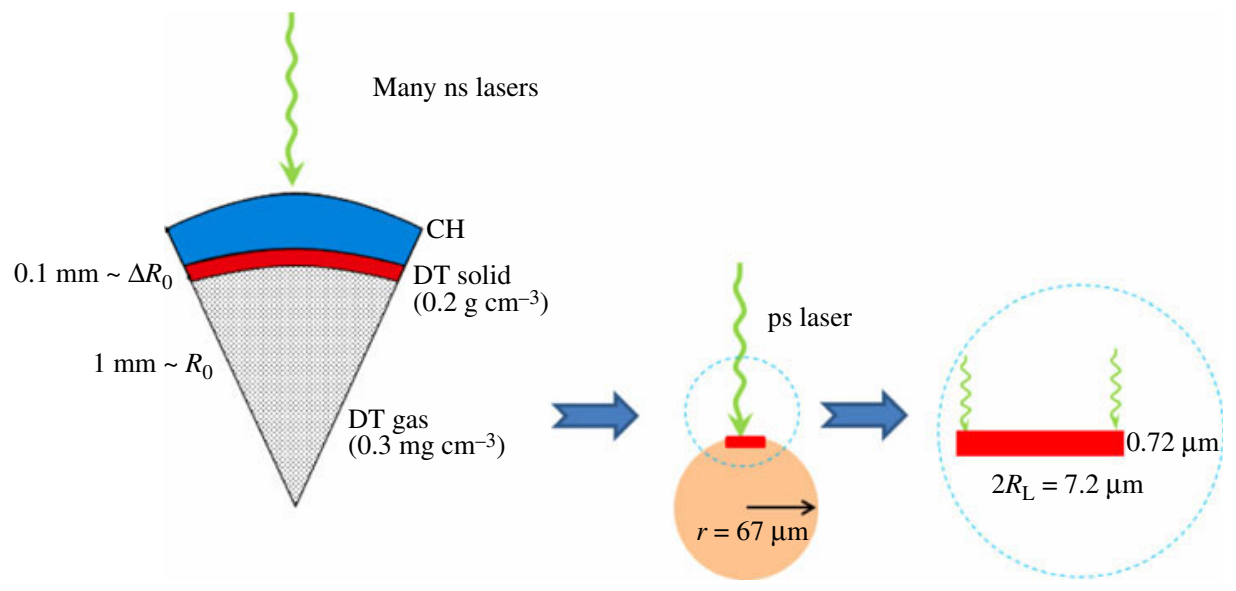

Figure 7. The FI scheme suggested in this paper. As a numerical example an initial pellet with radius $R_{0}=1 \mathrm{~mm}$ and DT fuel of density $0.2 \mathrm{~g} \mathrm{~cm}{ }^{-3}$ with thickness $0.1 \mathrm{~mm}$ (i.e., an aspect ratio of 10) is compressed to a density of $\rho_{0}=10^{3} \mathrm{~g} \mathrm{~cm}^{-3}$ by nanosecond lasers with a radius of $67 \mu \mathrm{m}$. The picosecond fast igniter laser with a $7.2 \mu \mathrm{m}$ beam diameter creates a shock wave pulse with a thickness of $0.72 \mu \mathrm{m}$ and can be considered a 1D shock wave to a reasonable approximation.

hydrodynamic instabilities, equations of state, nonlinear transport issues, non-local thermodynamic equilibrium, even neglecting energy conservation! (ii) Engineering simplicity. The IFE project is extremely complicated technologically and therefore a major effort is required in choosing the laser system, target design, etc., from all possible proposals by physicists. Technological simplicity must be seriously taken into account. For example, IFE requires $10^{8}$ or more laser shots per year; therefore complicated target designs (such as inserting a golden cone inside a pellet) are not realistic. (iii) Last, but not least, IFE is supposed to be economically practical. This implies the required gain, defined as the nuclear energy output divided by the laser input per shot, be larger than 100 and that the cost of a target should not be more expensive than 0.1 US\$.

Taking into account these three criteria it looks as though: (a) direct drive is simpler than indirect drive. (b) FI needs significantly less energy (approximately 0.3 MJ instead of $3 \mathrm{MJ}$ ). Therefore direct drive FI has the potential to be the best route to achieve nuclear fusion as an energy source. (c) From all presently known FI schemes the simplest FI seems to be by means of an 'extra shock' wave. We suggest a novel shock wave ignition scheme requiring less energy (in comparison with the present shock wave ignition scheme ${ }^{[25]}$ ) and free of laser-plasma instabilities (no more than $I_{\mathrm{L}} \lambda_{\mathrm{L}}^{2}=10^{14}\left(\mathrm{~W} \mathrm{~cm}^{-2}\right) \mu \mathrm{m}^{2}$ in the laser compression pulses). In this proposal the ignition shock wave is created by a high irradiance laser and the shock wave is induced by a ponderomotive force in the intermediate domain between the relativistic and non-relativistic hydrodynamics. For this case the relativistic shock wave formalism has to be considered as developed in our previous section. We call our scheme ultrafast, since the laser pulse duration for the ignition process is significantly smaller, by one to two orders of magnitude.
The shock wave ignition criteria for DT nuclear fuel are

$$
\begin{aligned}
& \text { (i) } \rho R^{\prime \prime}=\kappa \rho_{0}\left(u_{\mathrm{s}}-u_{\mathrm{p}}\right) \tau_{\mathrm{L}} \geqslant 0.3\left[\mathrm{~g} \mathrm{~cm}^{-2}\right] \\
& \text { (ii) } T \geqslant 10 \mathrm{keV} .
\end{aligned}
$$

For the DT fusion one has $A=2.5$ and $Z=1$, therefore equation (23) for $10 \mathrm{keV}$ temperatures and a compression of $\kappa=4$ implies a minimum dimensionless pressure $\Pi_{\min }=$ $3.4 \times 10^{-5}$. According to our solution the dimensionless laser irradiance satisfies $\Pi_{\mathrm{L}}>\Pi_{\mathrm{L}, \min }=1.8 \times 10^{-5} . \Pi_{\min }$ and $\kappa=4$ imply a minimum shock velocity and particle velocity $u_{\mathrm{s}} / c=0.59 \times 10^{-2}$ and $u_{\mathrm{p}} / c=0.44 \times 10^{-2}$ respectively. Using these values in equation $(25)_{(\mathrm{i})}$, one gets a laser pulse duration of $\tau_{\mathrm{L}}=1.6 \mathrm{ps}$. Assuming a precompression of $\rho_{\mathrm{o}}=10^{3} \mathrm{~g} \mathrm{~cm}^{-3}$, the $\Pi_{\mathrm{L}, \text { min }}=1.8 \times 10^{-5}$ requires $I_{\mathrm{L}}=4.8 \times 10^{22} \mathrm{~W} \mathrm{~cm}^{-2}$. The shock wave thickness turn out to be $l_{\mathrm{s}}=\left(u_{\mathrm{s}}-u_{\mathrm{p}}\right) \tau_{\mathrm{L}}=0.72 \mu \mathrm{m}$. In order to have a $1 \mathrm{D}$ shock wave to a reasonable approximation we require a laser focal spot radius $R_{\mathrm{L}}=5 l_{\mathrm{s}}$, implying a laser cross section of $S=\pi R_{\mathrm{L}}^{2}=4.0 \times 10^{-7} \mathrm{~cm}^{2}$. In this case the laser energy $W_{\mathrm{L}}$ and power $P_{\mathrm{L}}$ are $30 \mathrm{~kJ}$ and $19 \mathrm{PW}$ respectively. This example was taken to describe our concept in Figure 7. As a numerical example in this figure we take an initial pellet with radius $R_{0}=1 \mathrm{~mm}$ and a DT fuel of density $0.2 \mathrm{~g} \mathrm{~cm}^{-3}$ with thickness $0.1 \mathrm{~mm}$ (i.e., an aspect ratio of 10) that is compressed to a density of $\rho_{0}=10^{3} \mathrm{~g} \mathrm{~cm}^{-3}$ (with a radius of $67 \mu \mathrm{m})$ by the nanosecond lasers. The picosecond fast igniter laser with a $7.2 \mu \mathrm{m}$ in diameter creates a shock wave pulse with a thickness of $0.72 \mu \mathrm{m}$, which can be consider a $1 D$ shock wave to a reasonably good approximation. The compressed pellet has a radius much larger than $\sqrt{S} \gg l_{\mathrm{S}}$ in order to have a $1 \mathrm{D}$ shock wave. In Table 1 we show how larger values of $\Pi_{\mathrm{L}}$ change the laser and shock wave parameters.

The compression of a typical pellet as discussed in the literature $^{[12,47]}$ requires between 100 and $300 \mathrm{~kJ}$ of energy, 
Table 1. The laser is defined by its irradiance $I_{\mathrm{L}}$, pulse duration $\tau_{\mathrm{L}}$, energy $\mathrm{W}_{\mathrm{L}}$ and power $\mathrm{P}_{\mathrm{L}}$. This laser creates a shock wave with a compression $\kappa$ in a pre-compressed target with an initial density $\rho_{0}$. The shock wave thickness $\left(=\left(u_{\mathrm{S}}-u_{\mathrm{p}}\right) \tau_{\mathrm{L}}\right.$, where $u_{\mathrm{S}}$ and $u_{\mathrm{p}}$ are the shock wave velocity and the particle velocity respectively) and its cross section are $l_{\mathrm{S}}$ and $S$, respectively, satisfying $\sqrt{S} \gg l_{\mathrm{S}}$ in order to have a 1D shock wave.

\begin{tabular}{lcccccccc}
\hline$\Pi_{\mathrm{L}}$ & $\begin{array}{c}\rho_{0} \\
\left(\mathrm{~g} \mathrm{~cm}^{-3}\right)\end{array}$ & $\begin{array}{c}I_{\mathrm{L}} \\
\left.(\mathrm{W} \mathrm{cm})^{-2}\right)\end{array}$ & $\kappa$ & $\left(u_{\mathrm{S}}-u_{\mathrm{p}}\right) / c$ & $\begin{array}{c}\tau_{\mathrm{L}} \\
(\mathrm{ps})\end{array}$ & $\begin{array}{c}l_{\mathrm{s}} \\
(\mu \mathrm{m})\end{array}$ & $\begin{array}{c}S \\
\left(\mathrm{~cm}^{2}\right)\end{array}$ & $\begin{array}{c}W_{\mathrm{L}} \\
(\mathrm{kJ})\end{array}$ \\
\hline $1.8 \times 10^{-5}$ & $10^{3}$ & $4.8 \times 10^{22}$ & 4 & $0.15 \times 10^{-2}$ & 1.6 & 0.72 & $4.0 \times 10^{-7}$ & 30 \\
$(\mathrm{PW})$ & 19 \\
$1 \times 10^{-4}$ & $10^{3}$ & $2.7 \times 10^{23}$ & 4 & $0.5 \times 10^{-2}$ & 0.5 & 0.75 & $4.4 \times 10^{-7}$ & 60 \\
$1 \times 10^{-3}$ & $10^{3}$ & $2.7 \times 10^{24}$ & 4 & $1.3 \times 10^{-2}$ & 0.2 & 0.78 & $4.8 \times 10^{-7}$ & 260 \\
\hline
\end{tabular}

depending on the EOS, target design and the final required density. The FI in our case needs approximately $30 \mathrm{~kJ}$ of energy. Such a laser is under development and may be available in the near future.

\section{Summary and discussion}

Recently $^{[31]}$ it was suggested that relativistic shock waves with a shock wave velocity of more than $50 \%$ light speed can be created in the laboratory with HPLs which are recently under development. In this paper we discuss two novel ideas. The first is the transition domain between the relativistic and non-relativistic laser-induced shock waves. The second is the use of this transition domain for shockwave-induced ultrafast ignition of a pre-compressed target. The laser parameters for these purposes are calculated and the main advantages of this scheme are described. The many laser beams with few nanosecond pulses that compresses the target do not require $I_{\mathrm{L}} \lambda_{\mathrm{L}}^{2}=10^{15} \mathrm{~W} \mathrm{~cm}^{-2} \mu \mathrm{m}^{2}$ as in the previously proposed shock wave ignition scheme ${ }^{[25]}$, thus disturbing laser plasma instabilities do not occur. Furthermore, in the present scheme less energy is required in the main laser pulses where a picosecond laser with very high power $(\sim 30 \mathrm{PW})$ is required for the ultrafast ignition with the shock wave in the intermediate domain between the relativistic and non-relativistic hydrodynamics.

Presently existing petawatt lasers (see appendix B) might be used to start relativistic experimental research in the laboratory. Recent and future developments of HPLs in the multipetawatt domain could be important for relativistic shock waves in the laboratory with pressures of $10^{15}$ atmospheres or energy densities of the order of $10^{14} \mathrm{~J} \mathrm{~cm}^{-3}$. Such pressures or energy densities have been suggested so far only in astrophysical objects.

The ultrafast ignition scheme suggested in this paper appears advantageous in comparison with the many FI proposals, as given in our introduction section. It is based on the following merit criteria: (i) Understanding the physics, (ii) Engineering simplicity, and (iii) Economically practical. We think that shock wave FI is the best choice and the model suggested here between the relativistic and nonrelativistic domain has significant advantages and should be taken seriously into account.
Finally we must mention a very recent FI proposal ${ }^{[48]}$ using a laser system similar to our laser parameters estimated in Section 4. In particular this scheme requires a temporally tailored pulse with an energy of $65 \mathrm{~kJ}$ of duration $1.48 \mathrm{ps}$ with a maximum intensity of $4 \times 10^{22} \mathrm{~W} \mathrm{~cm}^{-2}$. This model is based on the use of a hole-boring ${ }^{[39,49,50]}$ phenomenon that enables the HPL beam to penetrate beyond the critical density. As early as 1971 it was analytically calculated ${ }^{[49]}$ that the condition for a laser to propagate in non-uniform plasma with an electron density $n_{\mathrm{e}}$ and density gradient scale length $L_{\mathrm{n}}$ beyond the electron critical density $n_{\mathrm{c}}$ is given by

$$
\begin{aligned}
& \frac{1}{4 \pi}\left(\frac{L_{\mathrm{n}}}{\lambda_{\mathrm{L}}}\right)\left(\frac{n_{\mathrm{c}}}{n_{\mathrm{e}}}\right)^{2} a^{2}>1 \\
& a \equiv \frac{e E_{\mathrm{L}} \lambda_{\mathrm{L}}}{2 \pi m_{\mathrm{e}} c^{2}} \approx 840\left[I_{\mathrm{L}} \lambda_{\mathrm{L}}^{2} / 10^{24}\left(\frac{W \cdot \mu \mathrm{m}^{2}}{\mathrm{~cm}^{2}}\right)\right]^{1 / 2} .
\end{aligned}
$$

PIC simulations ${ }^{[39,48,50]}$ derived $L_{\mathrm{n}} \sim 20 \lambda_{\mathrm{L}}$, implying a laser penetration up to approximately $10^{24} \mathrm{~cm}^{-3}$ for a one micron wavelength laser with an intensity $I_{\mathrm{L}}=5 \times$ $10^{22} \mathrm{~W} \mathrm{~cm}^{-2}$. The quasi mono-energetic ions ${ }^{[48]}$ generated by the tailored laser pulses penetrate beyond this density to ignite the pre-compressed pellet. The in situ accelerated ions are the driver of FI.

In our model the laser-induced relativistic shock wave induces the ignition. The preliminary compressed fusion target for ICF is usually spherically symmetric and the density increases very rapidly towards the core of the target when our shock wave model is applied as described in Figure 7. As long as the thickness of the shock wave $l_{\mathrm{S}}$ is much smaller than the density gradient $L_{\mathrm{n}}$, i.e., $L_{\mathrm{n}} \gg l_{\mathrm{s}}$, we can assume that the target is uniform in the shock wave domain. Looking at our Table 1 one gets $l_{\mathrm{S}} \sim 1 \mu \mathrm{m}$, which is much smaller than the density gradient $L_{\mathrm{n}} \sim 20 \lambda_{\mathrm{L}}$ derived in PIC simulations ${ }^{[48,50]}$.

The solution suggested in this paper, like all other solutions to the energy problem, is extremely difficult scientifically, and a lot of money and enormous optimism is required for a positive solution. HPLSE is complex, complicated but possible. If civilization is to survive we need large new sources of energy. To quote Mark Twain (1835-1910): 'And what is a man without energy? Nothing - nothing at all'. 


\section{Appendix A}

For convenience we write the nonrelativistic Hugoniot equations and the ideal gas EOS:

$$
\begin{aligned}
& \text { (i) } u_{\mathrm{p} 1}=\left[P_{1}-P_{0}\right]^{1 / 2}\left(\frac{1}{\rho_{0}}-\frac{1}{\rho_{1}}\right)^{1 / 2} \\
& \text { (ii) } u_{\mathrm{s}}=\left(\frac{1}{\rho_{0}}\right) \frac{\left[P_{1}-P_{0}\right]^{1 / 2}}{\left(\frac{1}{\rho_{0}}-\frac{1}{\rho_{1}}\right)^{1 / 2}} \\
& \text { (iii) } E_{1}-E_{0}=\left(\frac{1}{2}\right)\left[P_{1}+P_{0}\right]\left(\frac{1}{\rho_{0}}-\frac{1}{\rho_{1}}\right) \\
& \text { (iv) }\} E_{j}=\left(\frac{1}{\Gamma-1}\right)\left(\frac{P_{j}}{\rho_{j}}\right) \text { for } j=0,1 . \\
& \text { (v) }\}
\end{aligned}
$$

The equations are obtained from the relativistic equations (11) by using $e=\rho c^{2}+\rho E, P$ and $\rho E$ are much smaller than $\rho c^{2}$ and $v / c \ll 1$.

\section{Appendix B}

In this appendix we give a list of petawatt lasers that are in use in different laboratories at the end of the year 2013. The following data are not officially confirmed - however, this was used in the literature and conferences according to our knowledge.

USA

Michigan University, Ann Arbor: 10J/30fs

Texas University, Austin: 186J/167fs

Berkeley National Laboratory: 40J/40fs

Rochester University, Rochester: $1 \mathrm{~kJ} / 1 \mathrm{ps}$

LLNL, Livermore: $600 \mathrm{~J} / 500 \mathrm{fs}$

CHINA

Beijing National Laboratory: 32J/28fs

Shanghai Institute of Optics and Fine Mechanics: 35J/27fs

EUROPE

Central Laser Facility, UK: 500J/500fs \& 15J/30fs

Jena, Germany: 120J/120fs

GSI Darmstadt, Germany: 500J/500fs

JAPAN

Osaka University: 500J/500fs

KOREA

Gwangju University: 34J/30fs

\section{References}

1. J. H. Nuckolls, L. Wood, A. Thiessen, and G. B. Zimmermann, Nature 239, 139 (1972).

2. G. Velarde and N. Carpintero-Santamaria, Inertial Confinement Nuclear Fusion: A Historical Approach by its Pioneers (Foxwell and Davies Publ., 2007).
3. K. Mima, M. Murakami, S. Nakai, and S. Eliezer, Applications of Laser-Plasma Interactions S. Eliezer and K. Mima, eds. (CRC Press, Boca Raton, 2009).

4. J. M. Martinez Val, S. Eliezer, M. Piera, and G. Velarde, Phys. Lett. A 216, 142 (1996).

5. S. Eliezer and J. M. Martinez Val, Laser Part. Beams 16, 581 (1998).

6. S. Son and N. J. Fish, Phys. Lett. A 329, 76 (2004).

7. S. Eliezer, The Interaction of High Power Lasers with Plasmas (CRC Press, Boca Raton, 2002).

8. C. G. M. Van Kessel and R. Sigel, Phys. Rev. Lett. 33, 1020 (1974).

9. R. Cauble, D. W. Phillion, T. J. Hoover, N. C. Holmes, J. D. Kilkenny, and R. W. Lee, Phys. Rev. Lett. 70, 2102 (1993).

10. E. I. Moses, Nucl. Fusion 49, 104022 (2009).

11. J. D. Lindl, Inertial Confinement Fusion: The Quest for Ignition and High Gain Using Indirect Drive (Springer, New York, 1997).

12. S. Atzeni and J. Meyer-ter-Vehn, The Physics of Inertial Fusion (Clarendon Press, Oxford, 2004).

13. N. G. Basov, S. Y. Guskov, and L. P. Feoktistov, J. Sov. Laser Res. 13, 396 (1992).

14. M. Tabak, J. Hammer, M. E. Glinsky, W. L. Kruer, S. C. Wilks, J. Woodworth, E. M. Campbell, M. D. Perry, and R. J. Mason, Phys. Plasmas 1, 1626 (1994).

15. P. A. Norreys, R. Allot, R. J. Clarke, J. Colliers, D. Neely, S. J. Rose, M. Zepf, M. Santala, A. R. Bell, K. Krushelnick, A. E. Dangor, N. C. Woolsey, R. G. Evans, H. Habara, T. Norimatsu, and R. Kodama, Phys. Plasmas 7, 3721 (2000).

16. M. Roth, E. T. Cowan, M. H. Key, S. P. Hatchett, C. Brown, W. Fountain, J. Johnson, D. M. Pennington, R. A. Snavely, S. C. Wilks, K. Yasuike, H. Ruhl, F. Pegoraro, S. V. Bulanov, E. M. Campbell, M. D. Perry, and H. Powell, Phys. Rev. Lett. 86, 436 (2001).

17. R. Kodama, P. A. Norreys, K. Mima, A. E. Dangor, R. G. Evans, H. Fujita, Y. Kitagawa, K. Krushelnick, T. Miyakoshi, N. Miyanaga, T. Norimatsu, S. J. Rose, T. Shozaki, K. Shigemori, A. Sunahara, M. Tampo, K. A. Tanaka, Y. Toyama, and M. Zepf, Nature 412, 798 (2001).

18. J. M. Martinez Val and M. Piera, Fusion Tech. 32, 131 (1997).

19. A. Caruso and C. Strangio, Laser Part. Beams 19, 295 (2001).

20. S. Y. Guskov, Quantum Electron. 31, 885 (2001).

21. P. Lalousis, I. B. Foldes, and H. Hora, Laser Part. Beams 30, 233 (2012).

22. P. Lalousis, H. Hora, S. Eliezer, J. M. Martinez Val, S. Moustaizis, G. H. Miley, and G. Mourou, Phys. Lett. A 377, 885 (2013).

23. M. Murakami, H. Nagatomo, H. Azechi, F. Ogando, M. Perlado, and S. Eliezer, Nucl. Fusion 46, 9 (2006).

24. S. Eliezer, J. M. Martinez Val, and C. Deutsch, Laser Part. Beams 13, 43 (1995).

25. R. Betti, C. D. Zhou, K. S. Anderson, L. J. Perkins, W. Theobald, and A. A. Sokolov, Phys. Rev. Lett. 98, 155001 (2007).

26. S. Eliezer and J. M. Martinez Val, Laser Part. Beams 29, 175 (2011).

27. S. Eliezer and S. V. Pinhasi, High Power Laser Sci. Eng. 1, 44 (2013).

28. S. Jackel, D. Saltzmann, A. Krumbein, and S. Eliezer, Phys. Plasmas 26, 3138 (1983).

29. V. E. Fortov and I. V. Lomonosov, Shock Waves 20, 53 (2010).

30. S. Eliezer, Laser-Plasma Interactions and Applications, P. D. McKenna, P. D. Neely, R. Bingham and D. A. Jaroszynski, eds. 68th Scottish Universities Summer School in Physics, p. 49 (Springer Publication, Heidelberg, 2013). 
31. S. Eliezer, N. Nissim, E. Raicher, and J. M. Martinez Val, Laser Part. Beams 32, 243 (2014).

32. L. D. Landau and E. M. Lifshitz, Fluid Mechanics 2nd edition (Pergamon Press, Oxford, 1987).

33. A. H. Taub, Phys. Rev. 74, 3 (1948).

34. Y. B. Zeldovich and Y. P. Raizer, Physics of Shock Waves and High Temperature Hydrodynamic Phenomena (Academic Press Publications, New York, 1966).

35. S. Eliezer, A. Ghatak, and H. Hora, Fundamental of Equation of State (World Scientific, Singapore, 2002)

36. S. Eliezer and R. A. Ricci, High Pressure Equation of State: Theory and Application, Enrico Fermi School CXIII 1989, (North Holland Publication, Amsterdam, 1991).

37. S. Eliezer and H. Hora, Phys. Rep. 172, 339 (1989).

38. S. Eliezer, N. Nissim, J. M. Martinez Val, K. Mima, and H. Hora, Laser Part. Beams 32, 211 (2014).

39. N. Naumova, T. Schlegel, V. T. Tikhonchuk, C. Labaune, I. V. Sokolov, and G. Mourou, Phys. Rev. Lett. 102, 025002 (2009).

40. S. Eliezer, J. M. Martinez Val, and S. V. Pinhasi, Laser Part. Beams 31, 113 (2013).
41. T. Esirkepov, M. Borghesi, S. V. Bulanov, G. Mourou, and T. Tajima, Phys. Rev. Lett. 92, 175003 (2004).

42. H. Hora, P. Lalousis, and S. Eliezer, Phys. Rev. Lett. 53, 1650 (1984).

43. A. Di Piazza, C. Muller, K. Z. Hatsagortsyan, and C. H. Keitel, Rev. Modern Phys. 84, 1177 (2012).

44. R. Ruffini, G. Vereshchagin, and S. Xue, Phys. Rep. 487, 1 (2010).

45. S. Y. Guskov, Plasma Phys. Rep. 39, 1 (2013).

46. S. E. Bodner, A. J. Schmitt, and J. D. Sethian, High Power Laser Sci. Eng. 1, 2 (2013).

47. S. Eliezer, M. Murakami, and J. M. Martinez Val, Laser Part. Beams 25, 585 (2007).

48. S. M. Weng, M. Murakami, H. Azechi, J. W. Wang, N. Tasoko, M. Chen, Z. M. Sheng, P. Mulser, W. Yu, and B. F. Shen, Phys. Plasmas 21, 012705 (2014).

49. C. Max and F. Perkins, Phys. Rev. Lett. 20, 1342 (1971).

50. A. Pukhov and J. Meyer-ter-Vehn, Phys. Rev. Lett. 79, 2686 (1997). 